\title{
The influence of exposure to Toxoplasma Gondii on host lipid metabolism
}

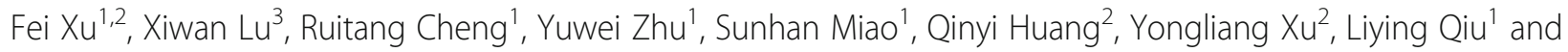
Yonghua Zhou ${ }^{2^{*}}$ (i)

\begin{abstract}
Background: Mounting evidence suggested a complex correlation between host lipid metabolism and Toxoplasma gondii (T. gondii) infection. However, the inherent association between T. gondii infection and host lipid state remains elusive either in mice or in human hosts.

Methods: Through a study in a sample of 1045 healthy participants from eastern China, we determined the association of T. gondii infection and host lipid levels using serological methods. We then examined the host lipid levels in C57BL/6 J mice at both acute and chronic T. gondii infection stages (for a period up to 36 weeks post infection).

Results: In our case-control study, T. gondii seropositive individuals had higher low-density lipoproteins (LDL) $(P=$ $0.0043)$ and total cholesterol (TC) $(P=0.0134)$ levels compared to seronegative individuals. Furthermore, individuals with $L D L(O R=3.25 ; 95 \% \mathrm{Cl}: 1.60-6.61)$ and TC (OR $=2.10 ; 95 \% \mathrm{Cl}: 1.22-3.63)$ levels above the upper limit of normal range had higher odds ratio to be $T$. gondii IgG positive. Consistently, in vivo data revealed that a significantly increased LDL level was first observed at early acute stage but plateaued to later time (chronic infection with $T$. gondii).

Conclusions: In both healthy population and T. gondii-infected mice, seropositive individuals had higher LDL level. Individuals with positive T. gondii IgG had more odds of being with LDL and TC abnormality. Latent T. gondii infection is common worldwide, potential medical interventions to host lipid metabolism may be a breakthrough point to the prevention and control of this parasite infection.
\end{abstract}

Keywords: Toxoplasma gondii, Parasitic infection, Triglycerides, Total cholesterol, High-density lipoproteins, Lowdensity lipoproteins

\section{Background}

Toxoplasma gondii ( $T$. gondii) is an obligate intracellular protozoan parasite. Currently, the estimated infection prevalence of T.gondii is relatively low in some countries, such as China (about 10\%), UK (about 10\%) and the USA (10 to 20\%) [1-3]. It can be over $40 \%$ in South

\footnotetext{
* Correspondence: toxo2001@163.com

${ }^{2}$ Key Laboratory of National Health Commission on Parasitic Disease Control and Prevention, Jiangsu Provincial Key Laboratory on Parasite and Vector Control, Jiangsu Institute of Parasitic Diseases and Public Health Research Center of Jiangnan University, Wuxi 214064, Jiangsu, China

Full list of author information is available at the end of the article
}

America and parts of Continental Europe [1]. T. gondii can infect almost all variety of warm-blooded animals. It invades and replicates in the intestinal system of hosts before entering the central nervous system. Mounting evidences show that $T$. gondii infection eventually triggers a wide range of clinical abnormalities, including miscarriage encephalitis [4, 5], Parkinson's disease [6], schizophrenia [7], obsessive-compulsive disorder [8] and Alzheimer's disease [9]. T. gondii transmits to humans mainly via the ingestion of food contaminated with cysts shed from bodily fluids or feces released by infected

C C The Author(s). 2020 Open Access This article is licensed under a Creative Commons Attribution 4.0 International License, which permits use, sharing, adaptation, distribution and reproduction in any medium or format, as long as you give appropriate credit to the original author(s) and the source, provide a link to the Creative Commons licence, and indicate if changes were made. The images or other third party material in this article are included in the article's Creative Commons licence, unless indicated otherwise in a credit line to the material. If material is not included in the article's Creative Commons licence and your intended use is not permitted by statutory regulation or exceeds the permitted use, you will need to obtain permission directly from the copyright holder. To view a copy of this licence, visit http://creativecommons.org/licenses/by/4.0/ The Creative Commons Public Domain Dedication waiver (http://creativecommons.org/publicdomain/zero/1.0/) applies to the data made available in this article, unless otherwise stated in a credit line to the data. 
carriers or from undercooked meat [10-13]. Primary infection in healthy individuals is usually asymptomatic, or occasionally generates mild influenza-like symptoms, accompanied by self-limiting lymphadenopathy and hepatosplenomegaly $[2,14]$. In immunosuppressed patients, T. gondii infection can lead to fatalities [15]. Given the prevalence of $T$. gondii in human beings, it is important to understand the parasite-induced physiological changes in mammals because of its potential farreaching clinical consequences.

$T$. gondii has an obligate intracellular existence indicating its reliance on a source of essential nutrients that can be obtained from the intracellular medium. Normal replication within the parasitophorous vacuole (PV) [16] of $T$. gondii requires considerable amounts of lipids for membrane biogenesis. Although T. gondii has autonomous capacity to synthesize phospholipids $[17,18]$, it still needs to convert the host's lipids intactly for membrane assembling due to the lack of the essential enzymes for sterols molecule synthesis [19]. A mobilization activity of host lipid resources to the parasites PV membrance (PVM) obviously exists in T. gondii [20, 21]. Owing to the profound effects of $T$. gondii on lipids in host, it is important to focus on the association between lipid levels and $T$. gondii infection in human subjects. However, studies about the T. gondii on the metabolism and uptake of lipids are not enough. So far only a few works have mentioned the association of $T$. gondii seropositivity with serum total cholesterol (TC) and high-density lipoprotein (HDL) levels in patients with schizophrenia [22].

Therefore, the correlation between the T. gondii seropositivity and serum lipid levels is poorly understood, especially in healthy individuals. A growing body of literatures have shown that lipid metabolic shifts occur in the host during acute $T$. gondii infection. However, the effect of these shifts has not been clarified in healthy population or in a chronic infection duration in vivo. This work is the first report revealing the association of T. gondii infection with serum TC, triglycerides (TG), low-density lipoprotein (LDL) and HDL levels, both in $T$. gondii infected mice model and a sample of healthy individuals.

\section{Methods}

\section{Inclusion criteria of participants}

We selected 1045 healthy participants from a physical examination population in Wuxi Affiliated Hospital of Nanjing Chinese Medicine University, Jiangsu Province, China. This study was performed from December 2018 to May 2019. Exclusion criteria for cases were as followed:1. Who didn't accept to participate in the study; 2. Who had taken cardiovascular abnormality, pathoglycemia or dyslipidemia control drugs in the past 3 months. 3. Individuals with advanced $T$. gondii-related diseases; 4. Individuals with cardiac, renal, liver insufficiency or other obvious abnormal physical symptoms; 5 . Individuals with severe mobility disorders or mental illness. The diagnosis of Toxoplasma gondii ( $T$. gondii) infection in selected individuals met the criteria of the $T$. gondii control manual. The randomly selected healthy participants included $89 \mathrm{~T}$. gondii seropositive individuals with 50 males and 39 females (average age of 31) and $956 \mathrm{~T}$. gondii seronegative individuals with 532 males and 424 females (average age of 39). Occupation or socioeconomic status was not restrictive criteria for enrollment. Our study was in accordance with the standards of the 1964 Declaration of Helsinki (as revised in Brazil 2013), and with approval of Wuxi Affiliated Hospital of Nanjing Chinese Medicine University, China (NO. 2018011715).

\section{Infection of mice}

A total of 120 female C57BL/6 J mice aged 6 weeks (SPF, weight median: $23.7 \mathrm{~g}$, range: 21.4 to $25.5 \mathrm{~g}$ ) in our experiments were purchased from the Comparative Medicine Center of Yangzhou University (experimental animal production license NO. SCXK-(su) 2012-0004). The mice were raised in the Animal Experiment Center of Jiangsu Institute of Parasite Prevention and Control (breeding environmental license number for experimental animals: SYXK-(su)2012-0034) and were housed in a humidity and temperature-controlled room on a light/ dark cycle of $12 \mathrm{~h}$, with free access to water and food. Mice were randomly divided into normal control and $T$. gondii infection group, with 60 mice in each group. Mice were orally infected with cysts suspension (including 6 to 8 cysts, isolated from the brains of mice infected with T. gondii two months post-infection) of Toxoplasma gondii Prugniaud (type II) strain. Mice in the normal control group were treated with normal saline by gavage. Five mice were dissected after anaesthetized by intraperitoneal injection of $1 \%$ sodium pentobarbital $(50 \mathrm{mg} / \mathrm{kg})$ at week $0,1,2,3,4,5,6,7,8,16,24$ and 36 p.i.. The study was carried out after obtaining ethical clearance from Ethical Review Board (Ref. No. IACUC-JIPD-2,016, 026) of Institute of Parasitic Disease Prevention and Control, Jiangsu, China.

\section{Lipids analysis}

Total cholesterol (TC) (normal range: $2.9-5.68 \mathrm{mmol} / \mathrm{L}$ ), high-density lipoprotein (HDL) (normal range: 0.9-2.19 $\mathrm{mmol} / \mathrm{L}$ ), low-density lipoprotein (LDL) (normal range: $1.9-3.6 \mathrm{mmol} / \mathrm{L}$ ) and triglycerides (TG) (normal range: 0.34-1.92 mmol/L) of human serum samples were measured on the Beckman Kurt AU5800 automatic biochemical analyzer, according to the manufacturer's recommendations. The range of normal lipid levels was 
set according to Guidelines for Prevention and Treatment of Dyslipidemia in Chinese Adults.

\section{Data analysis plan}

Data were analyzed using SPSS 18.0 (SPSS Inc. Chicago, Illinois). Descriptive statistics included standard deviations and proportions for continuous variables, proportions for categorical variables. Means were compared by student t-test. The association of $T$. gondii seropositive and different lipid indexes was analyzed with the rank sum test. We calculated the sample with a reference seroprevalence of 8 to $22 \%$ [23] as the expected frequency of exposure in T. gondii seropositive groups. We calculated the odds ratio (OR) and $95 \%$ confidence interval (CI). Statistical significance was set at a $P$ value $<0.05$.

\section{Results}

\section{T. Gondii seropositivity and serum lipid levels}

One thousand forty-five healthy individuals (mean age = $47.29 \pm 13.8$ years old) were enrolled in this study. The rate of $T$. gondii seropositivity in the entire sample was $8.51 \%$ (89/956), which was within the range of the estimated infection rate in China and several other countries [23-25]. Demographics and clinical features of the participant samples by anti- $T$. gondii IgG status (positive or negative) are presented in Table 1 . Male and female gender were basically evenly distributed in both $T$. gondii positive (male: $4.78 \%$, female:3.73\%) and negative groups (male:50.9\%, female:40.6\%) or in the entire sample (male: $55.7 \%$, female:44.3\%) (Table 1).

As demonstrated in Table 2, T. gondii seropositive individuals had nominally higher low-density lipoprotein (LDL) $(P=0.0043)$ and total cholesterol (TC) levels $(P=$ $0.0134)$. Triglycerides (TG) and high-density lipoprotein (HDL) levels, as well as ratio of TG to HDL, LDL to HDL and TC to HDL did not differ between $T$. gondii seropositive and seronegative individuals, neither in all samples, nor in samples with abnormal serum lipid levels (Table 2). As indicated in Table 3, individuals with TC $(P=0.024)$ and LDL $(P=0.010)$ beyond normal range had significant higher chance of being seropositive compared to those with normal range of lipid levels. However, individuals who were positive for anti-T. gondii IgG had approximately twice the odds of being LDL $(\mathrm{OR}=1.90 ; 95 \% \mathrm{CI}: 1.01-3.57 ; P<0.05)$ and TC $(\mathrm{OR}=$ 2.15; $95 \%$ CI: $1.27-3.64 ; P<0.05)$ abnormality compared to seronegative group (Table 3). It should be mentioned that, individuals with LDL $(\mathrm{OR}=3.25$; 95\% CI:1.60-6.61; $P<0.05)$ levels above the upper limit of normal range had even higher odds ratio to be $T$. gondii IgG positive. In addition, there was no significant difference in risks for T. gondii infection among individuals with different sex and age (Table 3).
Table 1 Demographics of study samples

\begin{tabular}{|c|c|c|c|c|}
\hline & \multicolumn{3}{|l|}{ T. gondii } & \multirow[t]{4}{*}{$p$ Value } \\
\hline & Seropositive & Seronegative & Combined & \\
\hline & $N=89$ & $N=956$ & $N=1045$ & \\
\hline & N (\%) & N (\%) & N (\%) & \\
\hline Sex & & & & 0.923 \\
\hline Male & $50(56.2)$ & $532(55.6)$ & $582(55.7)$ & \\
\hline Female & $39(43.8)$ & $424(44.4)$ & $463(44.3)$ & \\
\hline Age & & & & 0.794 \\
\hline$<40$ years & 28 (31.5) & $284(29.7)$ & $312(29.9)$ & \\
\hline 40 to $<50$ & $21(23.6)$ & $246(25.7)$ & 267 (25.6) & \\
\hline 50 to $<60$ & $26(29.2)$ & $246(25.7)$ & $272(26.0)$ & \\
\hline$\geq 60$ & $14(15.7)$ & 180 (18.8) & 194 (18.6) & \\
\hline TG & & & & 0.774 \\
\hline Low & 0 & 0 & 0 & \\
\hline Normal & $61(68.5)$ & $630(65.9)$ & $691(66.1)$ & \\
\hline High & $28(31.5)$ & $326(34.1)$ & 354 (33.9) & \\
\hline $\mathrm{HDL}$ & & & & 0.111 \\
\hline Low & $2(2.25)$ & $45(4.71)$ & $47(4.50)$ & \\
\hline Normal & 87 (97.8) & $911(95.3)$ & 998 (95.5) & \\
\hline High & 0 & 0 & 0 & \\
\hline LDL & & & & 0.01 \\
\hline Low & $2(2.25)$ & $40(4.18)$ & $42(4.02)$ & \\
\hline Normal & 76 (85.4) & 877 (91.7) & $953(91.2)$ & \\
\hline High & $11(12.4)$ & 39 (4.08) & $50(4.78)$ & \\
\hline $\mathrm{TC}$ & & & & 0.024 \\
\hline Low & $2(2.25)$ & $9(0.94)$ & $11(1.05)$ & \\
\hline Normal & $68(76.4)$ & $836(87.4)$ & $904(86.5)$ & \\
\hline High & $19(21.3)$ & 111 (11.6) & $130(12.4)$ & \\
\hline
\end{tabular}

Low: samples values exceed the lower limit of normal range; High: samples values exceed the upper limit of normal range; TG: triglycerides; HDL: high density lipoproteins; LDL: low density lipoproteins; TC: total cholesterol

Effect of T. gondii infection on weight and viscera of mice To further verify the influences of $T$. gondii infection on the lipid status in vivo, healthy C57BL/6 J mice were orally infected with 6 to 8 cysts by gavage. Acute response post-infection (p.i.) promptly occurred during week 2 to week 3 post-infection (p.i.). The weight curve $(n=6)$ declined to the lowest point at week 3 p.i., which recovered gradually thereafter. Significant decrease was found again at week $16,24,36$ p.i., possibly due to the deterioration of the host at late infection stage (Fig. 1a). In order to evaluate the effect of $T$. gondii infection on viscera, organ indexes were calculated. Cysts begin to form as early as one week after infection and persist in murine brain tissue for the rest of the host's life. The diameter of cysts increased during the latent infection stage in brains, eventually causing slightly but significantly higher 
Table 2 Lipid levels of study samples in T.gondii seropositive and seronegative groups

\begin{tabular}{llllll}
\hline & $\begin{array}{l}\text { SPG (All) } \\
(\mathrm{N}=89)\end{array}$ & $\begin{array}{l}\text { SNG (All) } \\
(\mathrm{N}=956)\end{array}$ & $\begin{array}{l}\text { Values out of limits } \\
\text { in SPG }\end{array}$ & $\begin{array}{l}\text { Values out of limits } \\
\text { in SNG }\end{array}$ & $\begin{array}{l}P \text { value } \\
(1 \mathrm{vs} .2,3 \mathrm{vs} .4)\end{array}$ \\
\hline TG $(\mathrm{mmol} / \mathrm{l})$ & $1.754 \pm 1.004$ & $1.909 \pm 1.637$ & $2.870 \pm 1.006(28)$ & $3.234 \pm 2.222(326)$ & $0.3795,0.3923$ \\
LDL $(\mathrm{mmol} / \mathrm{l})$ & $2.795 \pm 0.632$ & $2.612 \pm 0.573$ & $3.885 \pm 0.164(11)$ & $4.081 \pm 0.590(39)$ & $0.0043,0.2825$ \\
HDL $(\mathrm{mmo} / \mathrm{l})$ & $1.230 \pm 0.220$ & $1.190 \pm 0.211$ & - & - & $0.0888,-$ \\
TC $(\mathrm{mmol} / \mathrm{l})$ & $4.957 \pm 1.021$ & $4.714 \pm 0.872$ & $6.411 \pm 0.529(19)$ & $6.290 \pm 0.652(111)$ & $0.0134,0.4432$ \\
TG/HDL $(\mathrm{mmol} / \mathrm{l})$ & $1.534 \pm 1.096$ & $1.750 \pm 1.896$ & - & - & $0.2911,-$ \\
LDL/HDL $(\mathrm{mmol} / \mathrm{l})$ & $2.338 \pm 0.646$ & $2.249 \pm 0.576$ & - & - & $0.1700,-$ \\
TC/HDL $(\mathrm{mmol} / \mathrm{l})$ & $4.132 \pm 1.047$ & $4.060 \pm 0.968$ & - & - & $0.5074,-$ \\
\hline
\end{tabular}

SPG: T.gondii seropositive group; SNG: T.gondii seronegative group;

$1=$ T.gondii seropositive group (All); 2 = T.gondii seronegative group (All);

$3=$ Values out of limits in SPG; 4 = Values out of limits in SNG

All the values are formatted as: mean \pm SD

Table 3 Odds ratios for T.gondii seropositive participants compared to seronegative

\begin{tabular}{|c|c|c|}
\hline & \multicolumn{2}{|c|}{ T.gondii seropositive vs. seronegative } \\
\hline & OR & $95 \% \mathrm{Cl}$ \\
\hline \multicolumn{3}{|l|}{ Sex } \\
\hline Male & 1 & - \\
\hline Female vs. Male & 0.98 & $0.63,1.52$ \\
\hline \multicolumn{3}{|l|}{ Age } \\
\hline$<40$ years & 1 & - \\
\hline 40 to 50 vs. $<40$ & 0.87 & $0.48,1.56$ \\
\hline 50 to 60 vs. $<40$ & 1.07 & $0.61,1.88$ \\
\hline$\geq 60$ vs. $<40$ & 0.79 & $0.40,1.54$ \\
\hline \multicolumn{3}{|l|}{ TG } \\
\hline Normal & 1 & - \\
\hline Low vs. normal & - & - \\
\hline High vs. normal & 0.89 & $0.56,1.41$ \\
\hline \multicolumn{3}{|l|}{$\mathrm{HDL}$} \\
\hline Normal & 1 & - \\
\hline Low vs. normal & 0.47 & $0.11,1.95$ \\
\hline High vs. normal & - & - \\
\hline \multicolumn{3}{|l|}{ LDL } \\
\hline Normal & 1 & - \\
\hline Low vs. normal & 0.58 & $0.14,2.43$ \\
\hline High vs. normal & 3.25 & $1.60,6.61$ \\
\hline All vs. normal & 1.9 & $1.01,3.57$ \\
\hline \multicolumn{3}{|l|}{ TC } \\
\hline Normal & 1 & - \\
\hline Low vs. normal & 2.73 & $0.58,12.9$ \\
\hline High vs. normal & 2.1 & $1.22,3.63$ \\
\hline All vs. normal & 2.15 & $1.27,3.64$ \\
\hline
\end{tabular}

Low: samples values exceed the lower limit of normal range;

High: samples values exceed the upper limit of normal range;

TG: triglycerides; HDL: high density lipoproteins;

LDL: low density lipoproteins; TC: total cholesterol brain index in infected group at around week 5 p.i., as chronic stage began. Possibly due to the gradual severity of brain atrophy over time, a dramatically significant difference at week 36 p.i. occurred. T. gondii infection caused liver index rise to a certain extent at week 2 p.i. compared with control group (Fig. 1c), but conversely decreased at week 4 p.i.. Cysts transit to the tachyzoite stage within the digestive system and finally disseminate through the hosts' bloodstream to other tissues, once ingested by the host. Therefore, liver index increased at early stage post infection. Liver coefficient declined when cysts mainly metastasized to the brain, as approaching the chronic stage around week 5 p.i.. Perhaps due to immunosuppression at week 3 p.i., spleen index had a slight, albeit significant decrease in infected animals when compared to the control group (Fig. 1d).

Kinetics of serum lipids in mice during T. gondii infection We conducted continuous lipid levels monitoring during 1 to 36 weeks after T. gondii infection. As presented in Fig. 2, no difference between control and infected group was seen before week 24 p.i.. However, at week 36 p.i., the mean value of TG level decreased slightly in infected group (Fig. 2a). No significant variation at any time point was observed in HDL level (Fig. 2c). The alteration of TG/HDL ratio was reflected in the same kinetics as HDL level (Fig. 2e), which was not comparable between control and infected groups. TC level of infected group decreased sharply from week 1 p.i. Although it increased thereafter, but a small, albeit significant $(P=0.045)$ difference remained at week 4 p.i. as compared to control groups (Fig. 2b). Almost the same kinetics was observed in TC/HDL ratio as TC level (Fig. 2f). It was worth mentioning that, LDL level of infected animals increased sharply from week 2 to 5 p.i., which showed a significantly difference at week 5 p.i. when compared with control groups as the chronic infection 

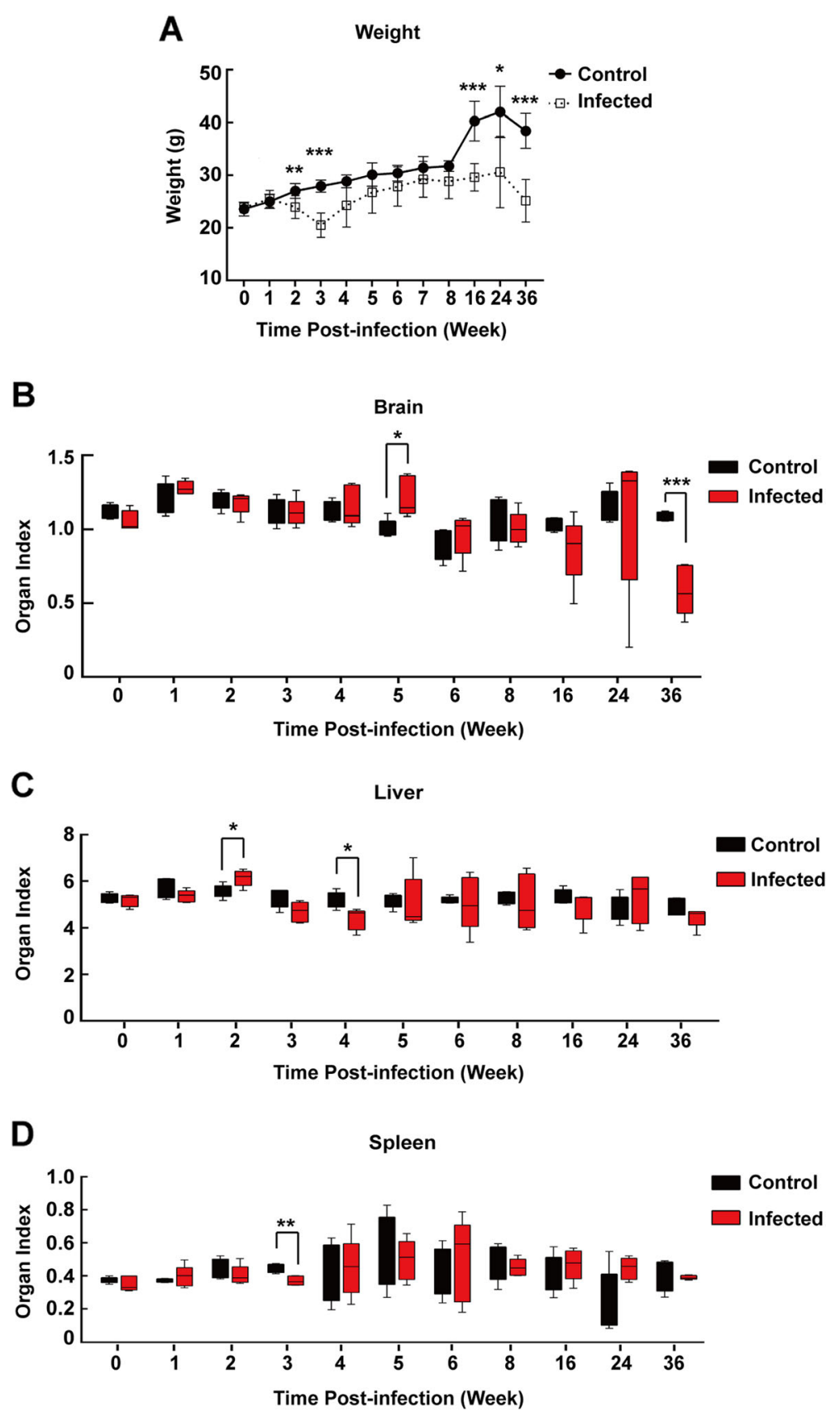

Fig. 1 Weight curve and relative organ index in T. gondii-infected C57BL/6 J mice at certain time point post-infection (p.i.). b brain, c liver, $\mathbf{d}$ spleen index: organ weight (g) / body weight $(\mathrm{g})(\mathbf{a})$. Values are expressed as mean \pm S.D. $(n=5)$. ${ }^{*} P<0.05$, ${ }^{* *} P<0.01,{ }^{* *} P<0.001$ compared with the normal control group

stage began. Although LDL level of infected group decreased thereafter from week 6 to week 16 p.i., a small but significantly increase occurred at week 24 p.i. (Fig. 2d). In Fig. 2g, LDL/HDL ratio in infected vs. control mice showed a trend similar to that in Fig. 2d: an increase occurred from week 5 to 6 p.i. but persisted comparable levels thereafter (Fig. 2g).

\section{Discussion}

It has been shown that the cholesterol of $T$. gondii parasitophorous vacuole membrance (PVM) derived from endocytosed low-density lipoprotein (LDL) of the host, which was not endogenous synthesized by the host cells [19]. However, the exact mechanism underlying cholesterol uptake by intravacuolar $T$. gondii has not been 


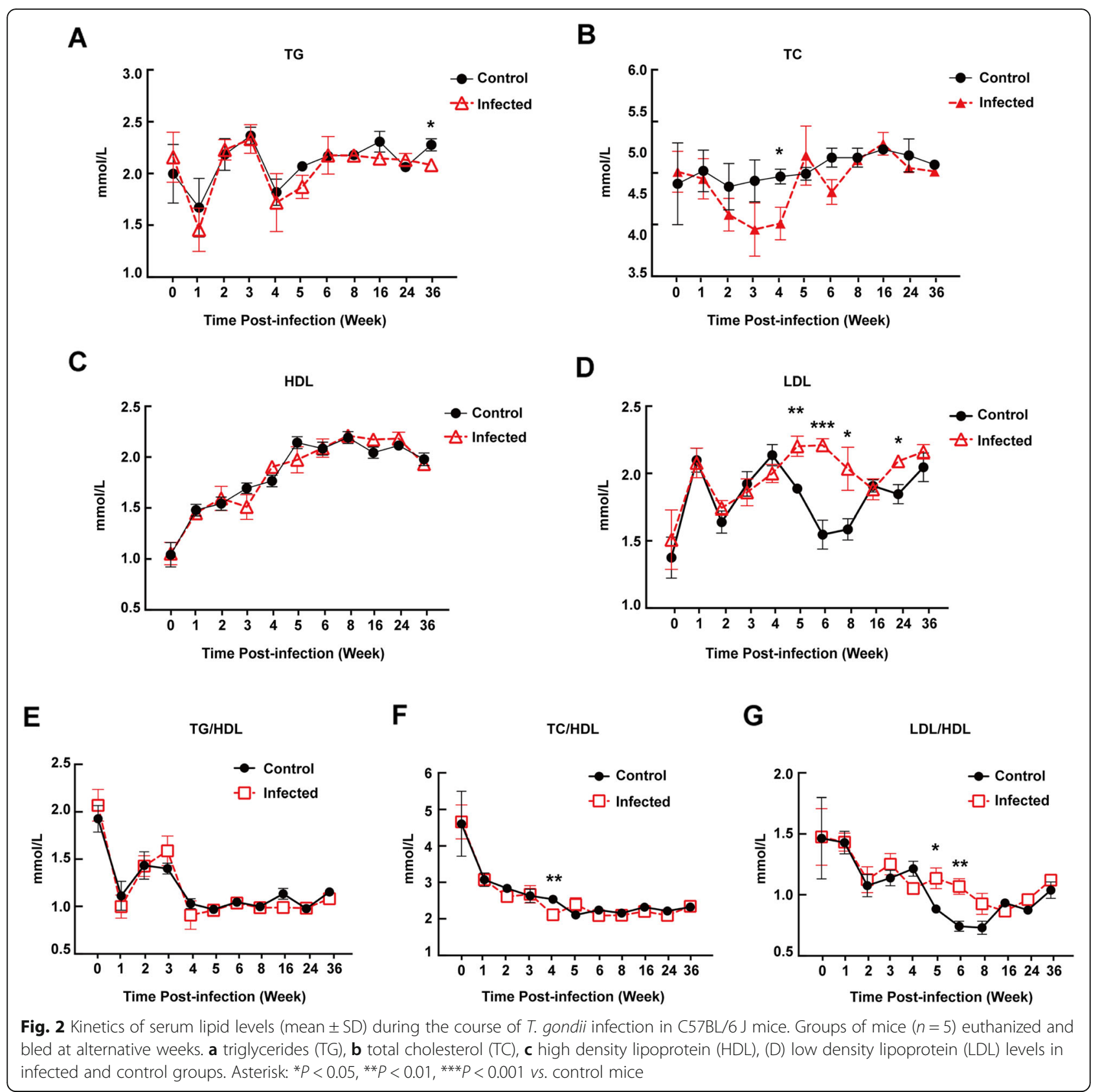

completely elucidated. To determine the influences of $T$. gondii infection on the host lipid status in vivo, 1045 healthy samples were randomly selected and carefully excluded by our inclusion criteria to assess the serum lipid levels. We demonstrated that abnormal total cholesterol (TC) and LDL levels were positively correlated with $T$. gondii seropositivity. Individuals whose serum TC level fell outside the normal range or LDL level exceeded upper limit of normal range had higher odds ratio for $T$. gondii infection. Consistent with the above observations, individuals with T. gondii antibodies had higher serum LDL and TC levels compared to T. gondii seronegative samples. However, the mean value of LDL and TC levels exceeded upper limit of normal range had no significant difference within groups. Further studies are necessary to clarify how $T$. gondii mobilize the host's lipid kinetics by a more systemic way. Thus, studies conducted in clinical samples rather than at cellular level are of critical importance.

As cholesterol in parasites is derived from LDL of the host, scavenging host lipids is critical for $T$. gondii survival [26]. Labeled fatty acid showed that T. gondii 
scavenge precursors from its host to synthesize full range of lipids [27]. However, a few studies about the association of $T$. gondii infection and host lipid metabolism showed obscure results [28-31]. Peer works reported that the only significant change in patients with schizophrenia was decreased serum triglyceride (TG) to high-density lipoprotein (HDL) ratio, observed in $T$. gondii seropositive group. Another study found no significant difference in TC level from cord blood serum between $T$. gondii seropositive and seronegative pregnant women [32]. Lipid metabolism in mice model with T. gondii infections is poorly understood. A following study showed that the major lipid metabolism alterations in mice included a decrease in serum HDL. TC level also decreased during acute phase (day 14 p.i.), which persisted until the end of the experiment (day 42 p.i.). An increase of LDL was observed at day 42 p.i., and no alterations in TG level were observed at any time point [33]. However, our results differed from the previous work, possibly due to the different mice (Swiss-Webster mice) or $T$. gondii strain (low virulence BGD-1 strain, human origin type-2) used in this work were not the same with ours. We demonstrated that the LDL level in infected animals increased as chronic phase began (week 5 to 6 p.i.). At this time point cysts formed and began to take advantage of the host-derived LDL. The increase of LDL was attributed to infection by $T$. gondii, as LDL is the major source from which $T$. gondii takes cholesterol [19]. To the contrary, serum TC levels decreased at day 7 p.i., at a time of established acute infection. Thus, we postulated that upon infection with $T$. gondii, uptake of cholesterol from serum LDL by rapidly multiplying tachyzoites triggered mobilization of cholesterol from the host's liver to the peripheral blood in the form of VLDL and LDL. A generally decreasing trend was therefore observed in the TC curve of infected group, although it increased at later time points. It should be noted that, wild-type inbred strain of mice have low serum LDL levels unlike ApoE- or $\mathrm{LDLr}^{-/-}$mice [34]. We postulated that was the reason why LDL levels lagged behind TC until the time of early chronic infection. Once LDL was mobilized to the periphery, it is utilized by rapidly proliferating tachyzoites, resulting in a constant LDL levels in the acute phase.

However, our studies had some limitations due to a relatively small cohort of subjects (1045 individuals) attending a local public hospital for physical examination. Moreover, this cross-sectional study did not allow the investigation on potential causality between host lipid metabolism and $T$. gondii, including a forward direction or vice versa. If abnormal lipid levels increased the risk of susceptibility to $T$. gondii? Or common causality, i.e., a shared factor causing both lipid abnormality and $T$. gondii susceptibility. Furthermore, we only demonstrated the physiological changes of host lipid levels after $T$. gondii infection but hadn't clarified if such changes were due to the need for parasites proliferation or the response of hosts to infection.

Despite these limitations, to our knowledge, this is the first study to identify the association between host lipid metabolism and $T$. gondii infection among healthy individuals. Positive serum IgG and "not too high" OD values are usually indicated in chronic infection stage. The chronic infection stage of mice, generally 5 weeks after infection, is to some extent comparable with that of human beings. Our results showed that during chronic infection stage, the serum lipid levels of mice infected with $T$. gondii had similarity with that of human, especially the LDL phenotype. Moreover, we successively investigated lipid metabolism kinetics and monitored as long as 36 weeks p.i. in the wild-type murine host. We considered that the host's lipid levels during acute or early chronic infection phase non-representative of the normal circumstances, since primary infection in healthy individuals was usually asymptomatic. In short, data presented here suggested that $T$. gondii had a potential effect on host lipid metabolism homeostasis during a long infection period. As host lipid metabolism is a complex process related to energy metabolism, immune system function etc., further studies are needed to clarify the mechanisms underlying this causality (i.e., whether abnormal lipid metabolism of the host to be the cause or effect to the risk of latent infection). Interventions targeting host lipid metabolism may be potential strategies for $T$. gondii infection since this parasite strictly rely on lipid metabolism of the host.

\section{Conclusion}

In this study, we investigated the association between $T$. gondii seroprevalence and serum lipid levels in healthy population. Furthermore, experimental mice were used as acute and chronic infection model to verify the dynamic influence of $T$. gondii on serum lipid in vivo. All these data suggested that $T$. gondii infection affected lipid levels in the host. Interventions targeting host lipid metabolism may be potential strategies for $T$. gondii infection since this parasite strictly rely on lipid metabolism of the host.

\section{Abbreviations \\ T. gondii: Toxoplasma gondii; p.i.: Post-infection; TG: Triglycerides; TC: Total cholesterol; LDL: Low density lipoproteins; HDL: High density lipoproteins; ACAT-1: Cholesterol acyltransferase 1; PV: Parasitophorous vacuole; OR: Odds ratio; $\mathrm{Cl}$ : Confidence interval}

\section{Acknowledgements}

We thank the general support of public research platform, Wuxi School of medicine, Jiangnan University and Jiangsu Institute of Parasitic Diseases and Public Health Research Center. We are grateful to Ze Sun, Junqi Yang, Xiuxia Qu for reviewing the English as well as for expert editorial assistance. 


\section{Authors' contributions}

FX, RT-C and QY-H performed the statistical analysis and drafted the manuscript, YH-Zh, XW-L and FX conceived and designed the study, SH-M and YW-Zh conducted the dates collection and chart making. YL-X, WY and LY-O write up of the proposal. YL-X, WY, SH-M, YW-Zh and LY-Q reviewed and edited the manuscript. All authors read and approved the final version of the paper

\section{Funding}

This work was supported by grants from Natural Science Foundation of Jiangsu Province (BK20190597), Wuxi Science and Technology Development Fund 2018 (Grant no. WX18IIAN010) and Jiangnan University Youth Fund 2018 (Grant no. K2050205), Chinese Postdoctoral Science Fund (Grant no. 2019 M661729), Health Project of Science and Education (Grant no. ZDXKA2016016), Medical Science and Technology Project of Health Commission of Jiangsu Province (Grant no. YB2017048). The authors also would like to extend sincere gratitude to the National First-Class Discipline Program of Food Science and Technology (JUFSTR20180101). The funding sources did not have a role in the design of the study and did not have any role during its data collection, analysis, interpretation of the data, or decision to submit results and writing of the manuscript.

\section{Availability of data and materials}

All the data supporting the study findings are within the manuscript. Additional detailed information will be shared upon request addressed to the corresponding author.

\section{Ethics approval and consent to participate}

The study was approved by the ethical review committee of the Animal Care and Use Committee at Jiangsu Institute of Parasitic Diseases (Ethics statement NO. IACUC-JIPD-2016026). Informed written consent was obtained from the subjects before collecting the stool samples and information.

\section{Consent for publication}

Not applicable.

\section{Competing interests}

The authors declare that they have no competing interests.

\section{Author details}

'Department of Basic Medicine, Wuxi Medical School, Jiangnan University, Wuxi, Jiangsu 214122, P.R. China. ${ }^{2}$ Key Laboratory of National Health Commission on Parasitic Disease Control and Prevention, Jiangsu Provincial Key Laboratory on Parasite and Vector Control, Jiangsu Institute of Parasitic Diseases and Public Health Research Center of Jiangnan University, Wuxi 214064, Jiangsu, China. ${ }^{3}$ Wuxi Hospital of Traditional Chinese Medicine, Wuxi Affiliated Hospital of Nanjing Chinese Medicine University, Wuxi, Jiangsu 214071, P.R. China.

\section{Received: 13 February 2020 Accepted: 4 June 2020}

\section{Published online: 15 June 2020}

\section{References}

1. Pappas G, Roussos N, Falagas ME. Toxoplasmosis snapshots: global status of toxoplasma gondii seroprevalence and implications for pregnancy and congenital toxoplasmosis. Int J Parasitol. 2009;39(12):1385-94.

2. Strharsky J, Klement C, Hruba F. Seroprevalence of toxoplasma gondi antibodies in the Slovak Republic. Folia Microbiol. 2009;54(6):553-8.

3. Yun F, Hui-Fang L, Min-Yuan S. Investigation on Toxoplasma gondii infection and awareness of toxoplasmosis-related knowledge in women with poor pregnant outcomes in Wuxi City. Zhongguo Xue Xi Chong Bing Fang Zhi Za Zhi. 2016;28(6):664-8

4. Shojaee S, Teimouri A, Keshavarz H, Azami SJ, Nouri S. The relation of secondary sex ratio and miscarriage history with toxoplasma gondii infection. BMC Infect Dis. 2018;18(1):307.

5. Khademi SZ, Ghaffarifar F, Dalimi A, Davoodian P, Abdoli A. Prevalence and risk factors of toxoplasma gondii infection among pregnant women in Hormozgan Province, south of Iran. Iran J Parasitol. 2019;14(1):167-73.

6. Zhou Z, Zhou R, Li K, Wei W, Zhang Z, Zhu Y, Luan R. The association between toxoplasma gondii infection and risk of Parkinson's disease: a systematic review and meta-analysis. Biomed Res Int. 2019;2019:8186017.
7. Chen X, Chen B, Hou X, Zheng C, Yang X, Ke J, Hu X, Tan F. Association between toxoplasma gondii infection and psychiatric disorders in Zhejiang, southeastern China. Acta Trop. 2019;192:82-6.

8. Nayeri Chegeni T, Sarvi S, Amouei A, Moosazadeh M, Hosseininejad Z, S AA Daryani A. Relationship between toxoplasmosis and obsessive compulsive disorder: a systematic review and meta-analysis. PLoS Negl Trop Dis. 2019; 13(4):e0007306

9. Nayeri Chegeni T, Sarvi S, Moosazadeh M, Sharif M, Aghayan SA, Amouei A, Hosseininejad Z, Daryani A. Is toxoplasma gondii a potential risk factor for Alzheimer's disease? A systematic review and meta-analysis. Microbial Pathogenesis. 2019;137:103751.

10. Montoya JG, Liesenfeld O. Toxoplasmosis. Lancet (London, England). 2004; 363(9425):1965-76.

11. Hide $\mathrm{G}$. Role of vertical transmission of toxoplasma gondii in prevalence of infection. Expert Rev Anti-Infect Ther. 2016;14(3):335-44.

12. Giakoumelou S, Wheelhouse N, Cuschieri K, Entrican G, Howie SE, Horne AW The role of infection in miscarriage. Hum Reprod Update. 2016;22(1):116-33.

13. Haq SZ, Abushahama MS, Gerwash O, Hughes JM, Wright EA, Elmahaishi MS, Lun ZR, Thomasson D, Hide G. High frequency detection of toxoplasma gondii DNA in human neonatal tissue from Libya. Trans R Soc Trop Med Hyg. 2016;110(9):551-7.

14. Krick JA, Remington JS. Toxoplasmosis in the adult--an overview. N Engl J Med. 1978:298(10):550-3

15. Robert-Gangneux F, Sterkers Y, Yera H, Accoceberry I, Menotti J, Cassaing S, Brenier-Pinchart MP, Hennequin C, Delhaes L, Bonhomme J, et al. Molecular diagnosis of toxoplasmosis in immunocompromised patients: a 3-year multicenter retrospective study. J Clin Microbiol. 2015;53(5):1677-84.

16. Coppens I. Contribution of host lipids to toxoplasma pathogenesis. Cell Microbiol. 2006:8(1):1-9.

17. Charron AJ, Sibley LD. Host cells: mobilizable lipid resources for the intracellular parasite toxoplasma gondii. J Cell Sci. 2002;115(Pt 15):3049-59.

18. Gupta N, Zahn MM, Coppens I, Joiner KA, Voelker DR. Selective disruption of phosphatidylcholine metabolism of the intracellular parasite toxoplasma gondii arrests its growth. J Biol Chem. 2005:280(16):16345-53.

19. Coppens I, Sinai AP, Joiner KA. Toxoplasma gondii exploits host low-density lipoprotein receptor-mediated endocytosis for cholesterol acquisition. J Cell Biol. 2000;149(1):167-80.

20. Foussard F, Leriche MA, Dubremetz JF. Characterization of the lipid content of toxoplasma gondii rhoptries. Parasitology. 1991;102(Pt 3):367-70.

21. Flegr J, Priplatova L, Hampl R, Bicikovia M, Ripova D, Mohr P. Difference of neuro- and immunomodulatory steroids and selected hormone and lipid concentrations between toxoplasma-free and toxoplasma-infected but not CMV-free and CMV-infected schizophrenia patients. Neuro Endocrinol Letters. 2014;35(1):20-7.

22. Sagud M, Vlatkovic S, Svob Strac D, Sviben M, Zivkovic M, Vilibic M, VuksanCusa B, Mihaljevic-Peles A, Pivac N. Latent toxoplasma gondii infection is associated with decreased serum triglyceride to high-density lipoprotein cholesterol ratio in male patients with schizophrenia. Compr Psychiatry. 2018;82:115-20.

23. Cox JJ, Slabach B, Hast JT, Murphy SM, Kwok OC, Dubey JP. High seroprevalence of toxoplasma gondii in elk (Cervus canadensis) of the Central Appalachians, USA. Parasitol Res. 2017;116(3):1079-83.

24. Dubey JP, Jones JL. Toxoplasma gondii infection in humans and animals in the United States. Int J Parasitol. 2008;38(11):1257-78.

25. Yang YR, Craig PS, Ito A, Vuitton DA, Giraudoux P, Sun T, Williams GM, Huang Z, Li Z, Wang Y, et al. A correlative study of ultrasound with serology in an area in China co-endemic for human alveolar and cystic echinococcosis. Tropical Med Int Health. 2007;12(5):637-46.

26. Bisanz C, Bastien O, Grando D, Jouhet J, Marechal E, Cesbron-Delauw MF. Toxoplasma gondii acyl-lipid metabolism: de novo synthesis from apicoplast-generated fatty acids versus scavenging of host cell precursors. Biochem J. 2006:394(Pt 1):197-205.

27. Sehgal A, Bettiol S, Pypaert M, Wenk MR, Kaasch A, Blader IJ, Joiner KA, Coppens I. Peculiarities of host cholesterol transport to the unique intracellular vacuole containing Toxoplasma. Traffic (Copenhagen, Denmark). 2005:6(12):1125-41.

28. Reeves GM, Mazaheri S, Snitker S, Langenberg P, Giegling I, Hartmann AM, Konte B, Friedl M, Okusaga O, Groer MW, et al. A Positive Association between T gondii Seropositivity and Obesity. Front Public Health. 2013;1:73.

29. Rubicz R, Leach CT, Kraig E, Dhurandhar NV, Grubbs B, Blangero J, Yolken R, Goring $\mathrm{HH}$. Seroprevalence of 13 common pathogens in a rapidly growing 
U.S. minority population: Mexican Americans from San Antonio, TX. BMC Res Notes. 2011;4:433.

30. Thjodleifsson B, Olafsson I, Gislason D, Gislason T, Jogi R, Janson C. Infections and obesity: a multinational epidemiological study. Scand J Infect Dis. 2008:40(5):381-6.

31. Wilking H, Thamm M, Stark K, Aebischer T, Seeber F. Prevalence, incidence estimations, and risk factors of toxoplasma gondii infection in Germany: a representative, cross-sectional, serological study. Sci Rep. 2016;6:22551

32. Sturchler D, Berger R, Just M. Congenital toxoplasmosis in Switzerland Seroprevalence, risk factors and recommendations for prevention. Schweiz Med Wochenschr. 1987;117(5):161-7.

33. Milovanovic I, Vujanic M, Klun I, Bobic B, Nikolic A, Ivovic V, Trbovich AM, Djurkovic-Djakovic O. Toxoplasma gondii infection induces lipid metabolism alterations in the murine host. Mem Inst Oswaldo Cruz. 2009;104(2):175-8.

34. Khovidhunkit W, Kim MS, Memon RA, Shigenaga JK, Moser AH, Feingold KR, Grunfeld C. Effects of infection and inflammation on lipid and lipoprotein metabolism: mechanisms and consequences to the host. J Lipid Res. 2004; 45(7):1169-96.

\section{Publisher's Note}

Springer Nature remains neutral with regard to jurisdictional claims in published maps and institutional affiliations.

Ready to submit your research? Choose BMC and benefit from:

- fast, convenient online submission

- thorough peer review by experienced researchers in your field

- rapid publication on acceptance

- support for research data, including large and complex data types

- gold Open Access which fosters wider collaboration and increased citations

- maximum visibility for your research: over $100 \mathrm{M}$ website views per year

At BMC, research is always in progress.

Learn more biomedcentral.com/submissions 